\title{
GROWTH IMPAIRED CHILDREN WITH EPIDERMOLYSIS BULLOSA HAVE INCREASED SERUM MARKERS OF INFLAMMATION AND REDUCED CIRCULATING IGF-1/IGFBP-3
}

\author{
A. Rao $^{1}$, J.E. Mellerio ${ }^{2}$, M. Sklar ${ }^{2}$, C. Brain ${ }^{2}$, J. Allgrove ${ }^{3}$, I.R. Sanderson ${ }^{1}$, A.E. Martinez ${ }^{2}$ \\ ${ }^{\text {I}}$ Centre for Digestive Diseases, Barts and the London School of Medicine and Dentistry, Queen Mary \\ University of London, ${ }^{2}$ Departments of Paediatric Dermatology and Endocrinology, Great Ormond Street \\ Hospital for Children NHS Trust, ${ }^{3}$ Department of Paediatric Endocrinology and Diabetes, Barts and the \\ London Children's Hospital, Royal London Hospital, London, UK
}

Background: Epidermolysis bullosa (EB) is a group of devastating diseases in which patients have skin inflammation with epithelial fragility and recurrent blistering. Children with EB have poor linear growth, which is only partially reversed by nutritional supplementation. Pro-inflammatory cytokines impair growth in children with inflammatory bowel disease by inhibiting signal transduction from growth hormone (GH) to insulin-like growth factor-1 (IGF-1). We hypothesised that a similar mechanism occurred in EB.

Objective: To examine growth impairment and IGF-1 levels in a cohort of patients with severe generalised recessive dystrophic EB (RDEB).

Methods: 18 children with RDEB were identified. Height, height velocity(HV) standard deviation scores(SDS), inflammatory markers and serum IGF-1 and IGF binding protein-3(IGFBP-3) were measured.

Results: The median age(range) of the children was 12.42 years(2.43-17.62). 10 were female, 8 male. All of the girls being premenarcheal, and the boys prepubertal. 17 of 18 had a negative height SDS (mean -2.43, SD 1.33) and 16 of 18 had negative HV SDS (mean -2.79, SD 2.51). The impaired growth occurred despite nutritional supplementation (16 of 18 had a gastrostomy in situ, 2 had oral supplementary feeds). All had elevated inflammatory markers (mean[SD] CRP 78.27[39.18], ESR 106.9[30.72]). All had low circulating IGF-1 (mean SDS -2.53[0.39]) and IGFBP-3 (mean SDS -2.48[0.68]). IGF-1 levels did not correlate to HV $\operatorname{SDS}(\mathrm{p}=0.58)$.

Conclusions: Children with severe generalised RDEB have poor growth and low circulating IGF-1 and IGFBP-3. This is likely to be due, in part, to inflammation. Correcting growth retardation will require nonsteroidal therapies targeted at reducing inflammation. 